
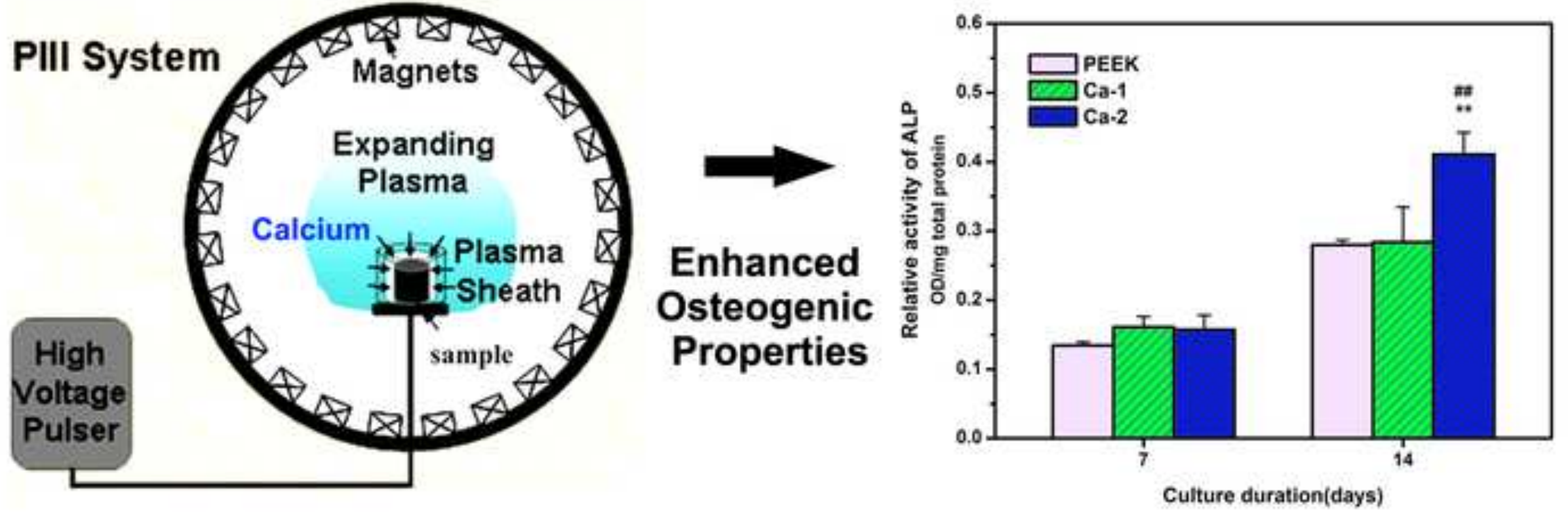


\title{
Enhanced osteogenic activity of poly ether ether ketone using calcium plasma immersion ion implantation
}

\author{
Tao Lu, Shi Qian, Fanhao Meng, Congqin Ning, Xuanyong Liu*
}

State Key Laboratory of High Performance Ceramics and Superfine Microstructure,

Shanghai Institute of Ceramics, Chinese Academy of Sciences, Shanghai 200050, China

\section{${ }^{*}$ Corresponding Author:}

Prof. Xuanyong Liu

State Key Laboratory of High Performance Ceramics and Superfine Microstructure,

Shanghai Institute of Ceramics, Chinese Academy of Sciences, Shanghai 200050,

China

E-mail: xyliu@mailsic.ac.cn

Tel: 86-21-52412409 
Abstract: As a promising implantable material, poly ether ether ketone (PEEK) possesses similar elastic modulus to that of cortical bones yet suffers from bio-inertness and poor osteogenic properties, which limits its application as orthopedic implants. In this work, calcium is introduced onto PEEK surface using calcium plasma immersion ion implantation (Ca-PIII). The results obtained from scanning electron microscopy (SEM) and X-ray photoelectron spectroscopy (XPS) confirm the modified layer with varying contents of calcium are formed on PEEK surfaces. Water contact angle measurements reveal the increasing hydrophobicity of both Ca-PIII treated surfaces. In vitro cell adhesion, viability assay, alkaline phosphatase activity and collagen secretion analyses disclose improved the adhesion, proliferation, and osteo-differentiation of rat bone mesenchymal stem cells (bMSCs) on Ca-PIII treated surfaces. The obtained results indicate that PEEK surface with enhanced osteogenic activity can be produced by calcium incorporation.

Key words: plasma immersion ion implantation; PEEK; calcium; osteogenic activity 


\section{Introduction}

Orthopedic implants have been increasingly required for impaired human bones caused by trauma, disease, aging or congenital defects [1]. Poly ether ether ketone (PEEK) is recently becoming a prime candidate to replace traditional metallic implants such as titanium and titanium alloys [2]. Despite of the good biocompatibility, titanium implants possess much higher elastic moduli (over $100 \mathrm{GPa}$ ), while PEEK has an adjustable elastic modulus close to that of cortical bone which can mitigate concerns over the risks of osteanabrosis and bone resorption caused by stress shielding as a result of the elasticity mismatch between the implants and human bones [3-5]. Besides, potential toxicity of ion release and declining mechanical properties of metallic implants caused by corrosion can be avoided due to the well known good chemical resistance of PEEK [3, 6, 7].

However, the excellent stability originated from the chemical structure makes PEEK bioinert, which impedes osteointegration after implantation thereby severely hampering clinical adoption as orthopedic implants $[2,3]$. To improve its osteogenic properties, calcium compounds have been introduced into PEEK in numerous ways, as calcium is one of the main constitutes in human bones. Moreover, studies have demonstrated that moderate amount of extracellular calcium ions plays a critical role in regulating proliferation and osteo-differentiation of osteoblasts and bone mesenchymal stem cells (bMSCs) [8]. For example, hydroxyapatite has been added into PEEK to improve bioactivity both in vitro and in vivo [9]. Poly ether ether ketone/ $\beta$-tricalcium phosphate (PEEK/ $\beta$-TCP) composites have also been developed to promote osteoblast 
proliferation [10]. Nevertheless, in contrast to the improved osteogenic properties, mechanical properties of PEEK compounds have been more or less sacrificed $[11,12]$.

During the healing process, surface properties play a crucial role in the early cell and bacterial behavior at the implant/tissue interface $[13,14]$. Therefore, surface modification offers an effective way to enhance the surface mechanical and biological properties meanwhile preserving the advantageous bulk properties of the materials. Among the various surface modification techniques, plasma immersion ion implantation (PIII) is a non-line-of-sight method that has been widely applied to microelectronics, aerospace engineering, precision manufacturing, and biomedical engineering [15-17]. By introducing different elements and functional groups, surface properties such as cytocompatibility, antibacterial activity, and mechanical properties can be selectively tailored [4, 18-22]. For instance, our previous work introduced silver nanoparticles on titanium by silver-PIII to enhance both biocompatibility and antibacterial activities [19]. Krupa et al implanted calcium and phosphorus onto titanium and improved the bioactivity and corrosion resistance [20].

Although a number of studies have already devoted to modifying titanium surface with PIII technique [19, 23, 24], surface modification of PEEK with PIII technique and the corresponding bMSC response to modified PEEK surface have been few studied $[25,26]$. In this work, $\mathrm{Ca}$ ions were implanted onto PEEK surface by calcium plasma immersion ion implantation (Ca-PIII). The effects of Ca-PIII process on the surface structure and surface composition were investigated. Furthermore, the osteogenic properties of the Ca-PIII modified PEEK surface were assessed in vitro using rat 
bMSCs.

\section{Materials and Methods}

\subsection{Sample preparation}

Biomedical grade poly ether ether ketone (PEEK) was used in this work. Square samples $\left(10 \times 10 \times 1 \mathrm{~mm}^{3}\right)$ were used for surface characterization, ion release tests and in vitro studies on 24-well tissue culture plates. The samples were one-side polished to a near mirror finish and ultrasonically cleaned in acetone, ethanol and ultra-pure water prior to PIII treatment. Calcium cathode rod was obtained from a large piece of $99.99 \%$ pure calcium bulk material and machined to the dimension of $10 \mathrm{~mm}$ diameter with $30 \mathrm{~mm}$ length. The calcium rod was kept in an inert environment to prevent oxidation before using. Table 1 lists the important parameters and the corresponding sample designation. Briefly, calcium was implanted into all samples using a magnetic filtered cathodic arc source housing the calcium rod. Before PIII process, the main chamber was vacuumed to a pressure of $5 \times 10^{-3} \mathrm{~Pa}$. PEEK samples were placed on the sample stage connected to high voltage. Calcium was controllably triggered, ionized and positively charged using a high-voltage pulsed source. By applying a pulsed negative high voltage, calcium ions were implanted and the sample stage was continuously rotated in order to obtain uniform ion implantation. 


\subsection{Surface characterizations}

\subsubsection{Surface structure and chemical characterizations}

The surface morphology of all samples was examined using field-emission scanning electron microscopy (FE-SEM, Hitachi S-4800, Japan) at different magnification without applying any conductive coating.

The surface chemical states were determined by X-ray photoelectron spectroscopy (XPS) (Physical Electronic PHI 5802 equipped with a monochromatic Al Ka source) in City University of Hong Kong.

To determine the surface wettability, static water contact angle was measured (Automatic Contact Angle Meter Model SL200B, Solon information technology Co., Ltd, China) by sessile drop method, using ultra-pure water as media. Each data was acquired from the average of three different areas on each sample.

\subsubsection{Ion release}

One piece of each sample was incubated in $10 \mathrm{~mL}$ phosphate buffered saline (PBS $1 \mathrm{M})$ for different immersion time $\left(7,14,28\right.$, and 56 days) at $37{ }^{\circ} \mathrm{C}$ without stirring. At a prescribed time, all the solution was withdrawn and analyzed using inductively-coupled plasma atomic emission spectroscopy (ICP-AES, JY2000-2, France) analysis to determine the amount of released calcium ions. The withdrawing solution was replaced with the same volume of fresh PBS. 


\subsection{In vitro studies}

\subsubsection{Cell culturing}

The bone mesenchymal stem cells (bMSCs, provided by Stem Cell Bank, Chinese Academy of Sciences, Shanghai, China) were isolated from the bone marrow of six-week-old male Fisher 344 rats. The bMSCs were cultured in the $\alpha$-minimum essential medium ( $\alpha$-MEM, Gibto-BRL, USA) with 10\% fetal bovine serum (FBS, Hyclone, USA), $1 \%$ antimicrobial of penicillin, and streptomycin at $37{ }^{\circ} \mathrm{C}$ in a humidified atmosphere of $5 \% \mathrm{CO}_{2}$. The $\alpha$-MEM was refreshed every 3 days during cell culturing. The experiments were carried out with the bMSCs before passage five. All the samples were sterilized with $75 \%$ alcohol for 3 hours and rinsed twice with sterile PBS before cell seeding.

\subsubsection{Cell proliferation and viability}

The alamarBlue ${ }^{\mathrm{TM}}$ (AbD Serotec Ltd, UK) assay was employed to quantitatively determine the cell proliferation and viability on the samples. The bMSCs were seeded on the samples (four replicates) on 24 -well plates at the density of $1 \times 10^{4}$ cells per well. After cell seeding for 24 hours, the culture medium was withdrawn. Followed by immersing in PBS twice for 10 min, all the samples were transferred to the new 24-well plates. After 1, 4 and 7 days, the culture medium was replaced by $0.5 \mathrm{~mL}$ of the fresh medium with $5 \%$ alamarBlue ${ }^{\mathrm{TM}}$ in each well. After incubation for $4 \mathrm{~h}, 100 \mu \mathrm{L}$ of the medium was transferred to a 96-well plate for measurement. The amount of reduced alamarBlue ${ }^{\mathrm{TM}}$ was determined by an enzyme-labeling instrument (BIO-TEK, ELX 800) at wavelengths of $570 \mathrm{~nm}$ and $600 \mathrm{~nm}$. The operation and calculation of cell 
proliferation followed the instruction of the alamarBlue ${ }^{\mathrm{TM}}$ assay.

\subsubsection{Alkaline phosphatase activity}

The bMSCs were seeded on the samples (four replicates) on 24-well plates at a density of $1 \times 10^{4}$ cells per well (cultured for 7 days) or $0.5 \times 10^{4}$ cells per well (cultured for 14 days). In the quantitative alkaline phosphatase (ALP) assay, after culturing for 7 and 14 days, a Bio-Rad protein assay kit (Bio-Rad, USA) was used to calculate the total protein content and the results were adjusted with a series of BSA (Sigma) standards by measuring the optical density (OD) values of the absorbance at $570 \mathrm{~nm}$. After incubation with p-nitrophenyl phosphate (Sigma) at $37{ }^{\circ} \mathrm{C}$ for $30 \mathrm{~min}$, the ALP activity was calculated and adjusted with a series of 4-Nitrophend $\mathrm{NaOH}(0.02 \mathrm{M})$ solutions by measuring the OD values at $405 \mathrm{~nm}$. The ALP levels were normalized to the total protein content and described as $\mu \mathrm{M} / \mathrm{mg}$ total proteins.

\subsubsection{Collagen secretion}

Collagen secretion of bMSCs on the samples was quantified using Sirius Red staining method. The bMSCs were seeded on the samples (four replicates) on 24-well plates at a density of $1 \times 10^{4}$ cells per well (cultured for 7 days) or $0.5 \times 10^{4}$ cells per well (cultured for 14 days). At a prescribed time, the samples were washed three times with PBS and fixed in $4 \%$ paraformaldehyde. Following rinses for three times in PBS, the samples were stained for collagen secretion using a $0.1 \%$ solution of Sirius Red (Sigma) in saturated picric acid for $18 \mathrm{~h}$. After washing with $0.1 \mathrm{M}$ acetic acid until the no more red color appearing, images were taken by laser scanning microscopy. In the quantitative analysis, the stain on samples was eluted in $1 \mathrm{~mL}$ of the destain solution 
(0.2 M NaOH/methanol=1:1). The optical density at $540 \mathrm{~nm}$ was measured using an enzyme-labeling instrument.

\subsection{Statistical analysis}

Statistically significant differences $(p)$ between groups were measured using the one-way analysis of the variance and Tukey's multiple comparison tests. All the statistical analyses were determined with the GraphPad Prism 5 statistical software package.

\section{Results}

\subsection{Characterization of surface}

The surface morphology of PEEK, Ca-1 and Ca-2 samples is shown in Fig. 1. The untreated PEEK (Fig. 1A) has a relatively flat and smooth surface except a few scratches caused by pre-polishing. After Ca-PIII, deposited films can be clearly seen covering the surface of Ca-1 sample, as shown in Fig. 1B. As the implantation voltage increases to $30 \mathrm{kV}$, small nanoparticles are observed emerging on the surface of $\mathrm{Ca}-2$ compared with $\mathrm{Ca}-1$, which is assumed to be caused by the much higher energetic ion bombardment.

The histogram of static water contact angles of all samples is presented as Fig. 2. The contact angle of untreated PEEK is $84.9 \pm 0.9^{\circ}$. After Ca-PIII treatment, the contact angles of Ca-1 and Ca-2 samples increases to $106.4 \pm 0.9^{\circ}$ and $108.4 \pm 0.4^{\circ}$, showing that the surface become more hydrophobic after Ca-PIII.

The XPS spectra from surfaces of all three sample groups are shown in Fig. 
3(A C). In comparison with the XPS full spectrum of PEEK (Fig. 3A), characteristic peak of $\mathrm{Ca} 2 \mathrm{p} 3 / 2$ at $347.5 \mathrm{eV}$ is detected on both Ca-1 and Ca-2 surfaces (Figs. 3B and 3C). The calculated atomic concentrations of calcium on Ca-1 and Ca-2 surfaces are $4.2 \%$ and $11.1 \%$, respectively, indicating that more calcium can be introduced onto the PEEK surface through applying higher implantation voltage. The amount of oxygen is higher on both Ca-PIII treated surfaces compared with that on untreated PEEK, demonstrating that calcium on PEEK surfaces will finally stabilize as oxidation state when exposing to air.

The ICP-AES test results of calcium ion release from $\mathrm{Ca}-1$ and $\mathrm{Ca}-2$ are shown in Fig. 3D, showing that the calcium can be sustainably released in a long period (tested for 56 days). Besides, the released calcium of $\mathrm{Ca}-2$ surface is higher than that of $\mathrm{Ca}-1$ surface, which is in accordance with the XPS results.

\subsection{Response of bMSCs}

The marrow-derived bone mesenchymal stem cells (bMSCs) possess critical properties that make them uniquely suited for bone tissue engineering applications and have been considered ideal cells for the evaluation of osteogenic properties of bone substitutes. The time-related proliferation of bMSCs on all samples is evaluated using alamarBlue $^{\mathrm{TM}}$ assay after culturing for 1,4 , and 7 days. The proliferation data and corresponding cell morphology is presented in Figs. 4 and 5. After culturing for 1 day, cell proliferation on $\mathrm{Ca}-2$ is statistically higher than that both on PEEK $(* * * p<0.001)$ and $\mathrm{Ca}-1\left({ }^{\# \# \#} p<0.001\right)$ while the proliferation on $\mathrm{Ca}-1$ shows no statistical difference 
compared to PEEK. As the culturing time extends to 7 days, the number of bMSCs on Ca-PIII samples grows much larger than that on PEEK and the difference in statistics becomes more significant $(* * p<0.01$ for $\mathrm{Ca}-1$ and $* * * p<0.001$ for $\mathrm{Ca}-2)$, indicating that Ca-PIII samples are more beneficial to bMSC proliferation and the surface with more calcium is favorable to the initial proliferation. In addition of cell proliferation, as is seen from Fig. 5, the bMSCs on Ca-1 and Ca-2 surfaces show more filopodia and lamellipodia extensions compared with that on PEEK surface after culturing for 1 day, demonstrating that the introduction of calcium will alter the initial cellular adherence.

The osteoblastic differentiation ability of bMSCs is critical to bone regeneration and alkaline phosphatase (ALP) is considered as an early marker of osteoblastic differentiation. In this work, the ALP expression level on Ca-PIII samples is slightly higher than that on PEEK after culturing for 7 days (Fig. 6). However, as the culturing time extends to 14 days, the ALP expression on Ca-2 surface is significantly up-regulated compared with both PEEK $\left({ }^{* *} p<0.01\right)$ and Ca-1 $\left({ }^{\#} p<0.01\right)$, demonstrating the long-term stimulating effects of the calcium on bMSC osteoblastic differentiation.

Collagen secretion quantified by the Sirius Red staining is shown in Fig. 7. According to Figs. 7(A F), more collagen is secreted on the Ca-PIII treated surface compared to that on the PEEK surface. Besides, on the Ca-2 surface, significantly denser collagen is deposited than the Ca-1 surface, especially after culturing for 14 days. According to the quantitative results shown in Fig. 7G, collagen deposition on both the Ca-PIII treated surfaces increased significantly $(* * * p<0.001)$ compared with that on 
the PEEK surface. Moreover, collagen deposition level on the Ca-2 surface is also significantly higher $\left({ }^{\# \# \#} p<0.001\right)$ than that on the Ca-1 surface, showing that collagen deposition is more active on the surface with higher amount of calcium.

\section{Discussion}

In this work, we employ a calcium plasma immersion ion implantation (Ca-PIII) method to produce calcium-rich surface on PEEK. To address the functionality of calcium, we conduct different high voltages during plasma implantation process and acquire PEEK surfaces with two different contents of calcium, as is confirmed by XPS

(Fig. 3). Static water contact angle measurements disclose that both Ca-PIII treated surfaces (designated as Ca-1 and Ca-2) turn more hydrophobic (Fig. 2) probably caused by composition alteration during Ca-PIII as the surface structures of PEEK, Ca-1 and Ca-2 are not clearly changed (Fig. 1). However, Ca-1 and Ca-2 surfaces possess the same wettability according to the statistics, indicating surface wettability may be independent of $\mathrm{Ca}$ content.

Bone mesenchymal stem cells (bMSCs) abundantly exist in bone marrows that can differentiate into osteoblastic lineage. In this work, bMSCs are used to evaluate the osteogenic properties of all surfaces. According to our results, after Ca-PIII, adhesion, proliferation (Fig. 4) of bMSCs are well improved, particularly on the Ca-2 surface. Cell adhesion and proliferation are correlated with the bone formation ability. Hence, improved bMSC adhesion and proliferation of Ca-PIII treated surfaces indicate potentially better bonding between bone tissue and implants. ALP is the early marker 
of osteoblastic differentiation [27]. A higher ALP expression of bMSCs on $\mathrm{Ca}-2$ surface can be found after culturing for 14 days, showing Ca-2 surface is more favorable to bone formation. Besides, collagen secretion is also enhanced by the addition of $\mathrm{Ca}$ onto the PEEK surface, which can also be ascribed to the Ca content.

It is acknowledged that the increase of extracellular $\mathrm{Ca}$ ion will regulate cell proliferation and differentiation [8, 28]. Moreover, advanced and enhanced osteoblastic differentiation would occur in the presence of appropriate $\mathrm{Ca}$ ion concentration [29]. In addition, Ca ions have been proven to promote the expression of bone-associated proteins and collagen $[30,31]$. In this respect, we have monitored the amount of $\mathrm{Ca}$ ion released from both surfaces for 8 weeks (Fig. 3D). Our data disclose that more $\mathrm{Ca}$ ions are released from the $\mathrm{Ca}-2$ surface, which is up to $4.7 \mathrm{ppm}$ and triple the amount of $\mathrm{Ca}$ ions released from the $\mathrm{Ca}-1$ surface. Therefore, the enhanced osteogenic properties of $\mathrm{Ca}-2$ surface may be ascribed to the higher $\mathrm{Ca}$ ion concentration around the surface, which effectively stimulate bMSC functions and up-regulate the gene and protein expression of bMSCs.

\section{Conclusions}

Calcium incorporated PEEK surfaces are prepared by calcium plasma immersion ion implantation. The Ca-PIII treated surfaces become hydrophobic compared with untreated PEEK while showing independence on the content of calcium in this work. In vitro cell adhesion, viability assay, alkaline phosphatase activity and collagen secretion of bMSCs on the Ca-PIII surface are well promoted. Therefore, the surface 
osteogenic properties of PEEK are improved by calcium plasma immersion ion implantation and the application of plasma treated PEEK to orthopedic and dental implants could be expected to be broadened and expedited.

\section{Acknowledgments}

Financial support from the National Basic Research Program of China (973 Program, 2012CB933600), National Natural Science Foundation of China (81271704), Shanghai Committee of Science and Technology, China (14JC1493100 and 14XD1403900) are acknowledged. 


\section{Reference}

[1] M. Navarro, A. Michiardi, O. Castano, J.A. Planell, Biomaterials in orthopaedics, J.

R. Soc. Interface, 5 (2008) 1137-1158. 10.1098/rsif.2008.0151

[2] K.B. Sagomonyants, M.L. Jarman-Smith, J.N. Devine, M.S. Aronow, G.A. Gronowicz, The in vitro response of human osteoblasts to polyetheretherketone (PEEK) substrates compared to commercially pure titanium, Biomaterials, 29 (2008) 1563-1572. 10.1016/j.biomaterials.2007.12.001

[3] S.M. Kurtz, J.N. Devine, PEEK biomaterials in trauma, orthopedic, and spinal implants, $\quad$ Biomaterials, $28 \quad$ (2007) 4845-4869. DOI: 10.1016/j.biomaterials.2007.07.013

[4] H. Wang, M. Xu, W. Zhang, D.T.K. Kwok, J. Jiang, Z. Wu, P.K. Chu, Mechanical and biological characteristics of diamond-like carbon coated poly aryl-ether-ether-ketone, $\quad$ Biomaterials, $\quad 31 \quad$ (2010) 8181-8187. 10.1016/j.biomaterials.2010.07.054

[5] L.M. McNamara, J.C. Van der Linden, H. Weinans, P.J. Prendergast, Stress-concentrating effect of resorption lacunae in trabecular bone, J. Biomech., 39 (2006) 734-741. 10.1016/j.jbiomech.2004.12.027

[6] A. Katzer, H. Marquardt, J. Westendorf, J.V. Wening, G. von Foerster, Polyetheretherketone - cytotoxicity and mutagenicity in vitro, Biomaterials, 23 (2002) 1749-1759.

[7] J.M. Toth, M. Wang, B.T. Estes, J.L. Scifert, H.B. Seim, A.S. Turner, Polyetheretherketone as a biomaterial for spinal applications, Biomaterials, 27 (2006) 
324-334. 10.1016/j.biomaterials.2005.07.011

[8] E.M. Brown, R.J. MacLeod, Extracellular calcium sensing and extracellular calcium signaling, Physiological Reviews, 81 (2001) 239-297.

[9] K.L. Wong, C.T. Wong, W.C. Liu, H.B. Pan, M.K. Fong, W.M. Lam, W.L. Cheung, W.M. Tang, K.Y. Chiu, K.D.K. Luk, W.W. Lu, Mechanical properties and in vitro response of strontium-containing hydroxyapatite/polyetheretherketone composites, Biomaterials, 30 (2009) 3810-3817. DOI: 10.1016/j.biomaterials.2009.04.016

[10] L. Petrovic, D. Pohle, H. Munstedt, T. Rechtenwald, K.A. Schlegel, S. Rupprecht, Effect of beta TCP filled polyetheretherketone on osteoblast cell proliferation in vitro, Journal of Biomedical Science, 13 (2006) 41-46. 10.1007/s11373-005-9032-Z

[11] M.S. Abu Bakar, P. Cheang, K.A. Khor, Mechanical properties of injection molded hydroxyapatite-polyetheretherketone biocomposites, Composites Science and Technology, 63 421-425. Doi: 10.1016/s0266-3538(02)00230-0

[12] M.S. Abu Bakar, M.H.W. Cheng, S.M. Tang, S.C. Yu, K. Liao, C.T. Tan, K.A. Khor, P. Cheang, Tensile properties, tension-tension fatigue and biological response of polyetheretherketone-hydroxyapatite composites for load-bearing orthopedic implants, Biomaterials, 24 (2003) 2245-2250. Doi: 10.1016/s0142-9612(03)00028-0

[13] B.D. Boyan, T.W. Hummert, D.D. Dean, Z. Schwartz, Role of material surfaces in regulating bone and cartilage cell response, Biomaterials, 17 (1996) 137-146. $10.1016 / 0142-9612(96) 85758-9$

[14] X.Y. Liu, P.K. Chu, C.X. Ding, Surface nano-functionalization of biomaterials, Mater. Sci. Eng. R-Rep., 70 (2010) 275-302. 10.1016/j.mser.2010.06.013 
[15] P.K. Chu, C. Chan, Applications of plasma immersion ion implantation in microelectronics -- a brief review, Surface and Coatings Technology, 136 (2001) 151-156. Doi: 10.1016/s0257-8972(00)01046-X

[16] X.B. Tian, P.K. Chu, R. Fu, S.Q. Yang, Hybrid processes based on plasma immersion ion implantation: a brief review, Surface and Coatings Technology, 186 (2004) 190-195. DOI: 10.1016/j.surfcoat.2004.04.049

[17] P.K. Chu, Plasma surface treatment of artificial orthopedic and cardiovascular biomaterials, Surface \& Coatings Technology, $201 \quad$ (2007) 5601-5606. 10.1016/j.surfcoat.2006.07.003

[18] H. Wang, D.T.K. Kwok, W. Wang, Z. Wu, L. Tong, Y. Zhang, P.K. Chu, Osteoblast behavior on polytetrafluoroethylene modified by long pulse, high frequency oxygen plasma immersion ion implantation, Biomaterials, 31 (2010) 413-419. 10.1016/j.biomaterials.2009.09.066

[19] H. Cao, X. Liu, F. Meng, P.K. Chu, Biological actions of silver nanoparticles embedded in titanium controlled by micro-galvanic effects, Biomaterials, 32 (2011) 693-705. 10.1016/j.biomaterials.2010.09.066

[20] D. Krupa, J. Baszkiewicz, J.A. Kozubowski, A. Barcz, J.W. Sobczak, A. Bilinski, M. Lewandowska-Szumiel, B. Rajchel, Effect of dual ion implantation of calcium and phosphorus on the properties of titanium, Biomaterials, 26 (2005) 2847-2856. DOI: 10.1016/j.biomaterials.2004.08.015

[21] G. Jin, H. Cao, Y. Qiao, F. Meng, H. Zhu, X. Liu, Osteogenic activity and antibacterial effect of zinc ion implanted titanium, Colloids and Surfaces 
B-Biointerfaces, 117 (2014) 158-165. 10.1016/j.colsurfb.2014.02.025

[22] G. Jin, H. Qin, H. Cao, S. Qian, Y. Zhao, X. Peng, X. Zhang, X. Liu, P.K. Chu, Synergistic effects of dual $\mathrm{Zn} / \mathrm{Ag}$ ion implantation in osteogenic activity and antibacterial ability of titanium, Biomaterials, $35 \quad$ (2014) 7699-7713. 10.1016/j.biomaterials.2014.05.074

[23] P.K. Chu, Recent developments and applications of plasma immersion ion implantation, Journal of Vacuum Science \& Technology B, 22 (2004) 289-296. $10.1116 / 1.1632920$

[24] X.Y. Liu, R.W.Y. Poon, S.C.H. Kwok, P.K. Chu, C.X. Ding, Structure and properties of Ca-plasma-implanted titanium, Surface \& Coatings Technology, 191 (2005) 43-48. 10.1016/j.surfcoat.2004.08.118

[25] T. Lu, X. Liu, S. Qian, H. Cao, Y. Qiao, Y. Mei, P.K. Chu, C. Ding, Multilevel surface engineering of nanostructured $\mathrm{TiO} 2$ on carbon-fiber-reinforced polyetheretherketone, $\quad$ Biomaterials, $\quad 35 \quad$ (2014) 5731-5740. 10.1016/j.biomaterials.2014.04.003

[26] T. Lu, J. Wen, S. Qian, H. Cao, C. Ning, X. Pan, X. Jiang, X. Liu, P.K. Chu, Enhanced osteointegration on tantalum-implanted polyetheretherketone surface with bone-like elastic modulus, Biomaterials, $51 \quad$ (2015) 173-183. 10.1016/j.biomaterials.2015.02.018

[27] Y. Liu, P.R. Cooper, J.E. Barralet, R.M. Shelton, Influence of calcium phosphate crystal assemblies on the proliferation and osteogenic gene expression of rat bone marrow stromal cells, Biomaterials, $28 \quad$ (2007) 1393-1403. 
10.1016/j.biomaterials.2006.11.019

[28] Y.T. Sul, C. Johansson, T. Albrektsson, Which surface properties enhance bone response to implants? Comparison of oxidized magnesium, TiUnite, and osseotite implant surfaces, Int. J. Prosthodont., 19 (2006) 319-328.

[29] Y.-T. Sul, E.-s. Byon, Y. Jeong, Biomechanical measurements of calcium-incorporated oxidized implants in rabbit bone: effect of calcium surface chemistry of a novel implant, Clinical implant dentistry and related research, 6 (2004) 101-110. 10.1111/j.1708-8208.2004.tb00032.x

[30] P. Ducheyne, Q. Qiu, Bioactive ceramics: the effect of surface reactivity on bone formation and bone cell function, Biomaterials, 20 (1999) 2287-2303. $10.1016 / \mathrm{s} 0142-9612(99) 00181-7$

[31] Y.D.C. Halvorsen, D. Franklin, A.L. Bond, D.C. Hitt, C. Auchter, A.L. Boskey, E.P. Paschalis, W.O. Wilkison, J.M. Gimble, Extracellular matrix mineralization and osteoblast gene expression by human adipose tissue-derived stromal cells, Tissue Engineering, 7 (2001) 729-741. 10.1089/107632701753337681 


\section{Figure captions:}

Figure 1. Surface morphology of PEEK (A), Ca-1 (B) and Ca-2 (C).

Figure 2. Histogram of static water contact angles of PEEK, Ca-1 and Ca-2 samples.

Figure 3. XPS full spectra of PEEK (A), Ca-1 (B) and Ca-2 (C), and the Ca concentration of samples with immersion in 1M PBS solutions for various days (D).

Figure 4. Reduction percentage of alamarblue ${ }^{\mathrm{TM}}$ for bMSCs cultured on all samples for 1, 4 and 7 days. The statistical significance indicated by $* * p<0.01$ and $* * * p<0.001$ compared with PEEK while ${ }^{\# \#} p<0.01$ and ${ }^{\# \# \#} p<0.001$ compared with Ca-1.

Figure 5. SEM morphology of the bMSCs cultured on all samples for 1, 4, and 7 days.

Figure 6. ALP activity assay of the bMSCs cultured on all samples for 7 and 14 days. The statistical significance indicated by ${ }^{* *} p<0.01$ compared with PEEK while ${ }^{\# \#} p<0.01$ compared with Ca-1.

Figure 7. Optical images of collagen secretion by bMSCs cultured on PEEK (A), Ca-1 (B) and Ca-2 (C) for 7 days, on PEEK (D), Ca-1 (E) and Ca-2 (F) for 14 days. (G) Colorimetrically quantitative analysis of collagen secretion of all samples. The 
statistical significance indicated by $* * * p<0.001$ compared with PEEK while ${ }^{\# \# \#} p<0.01$ compared with Ca-1 
(A) PEEK

$400 \mathrm{~nm}$

(B) $C a-I$

(C) $\mathrm{Ca}-2$

$400 \mathrm{~nm}$
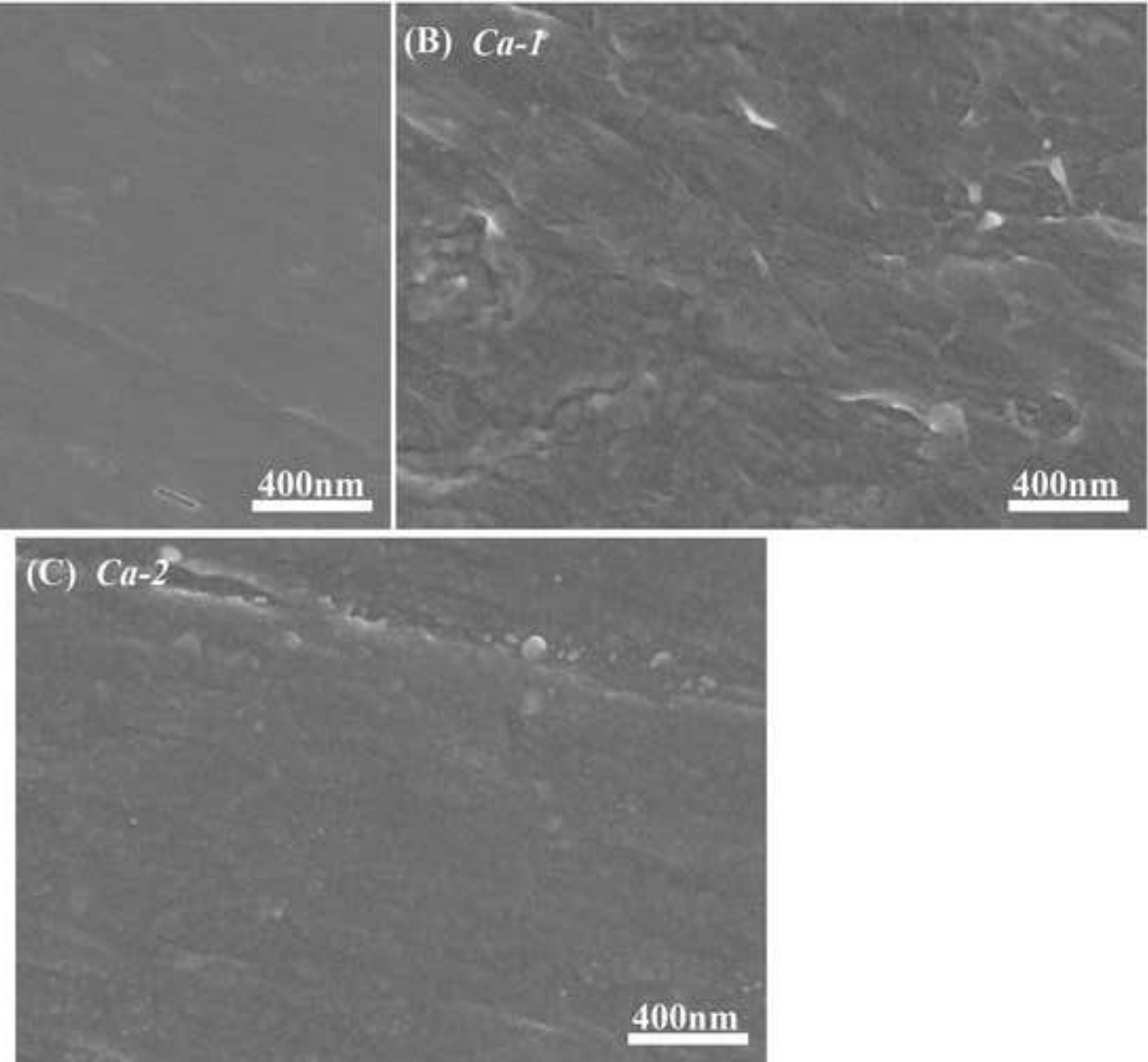

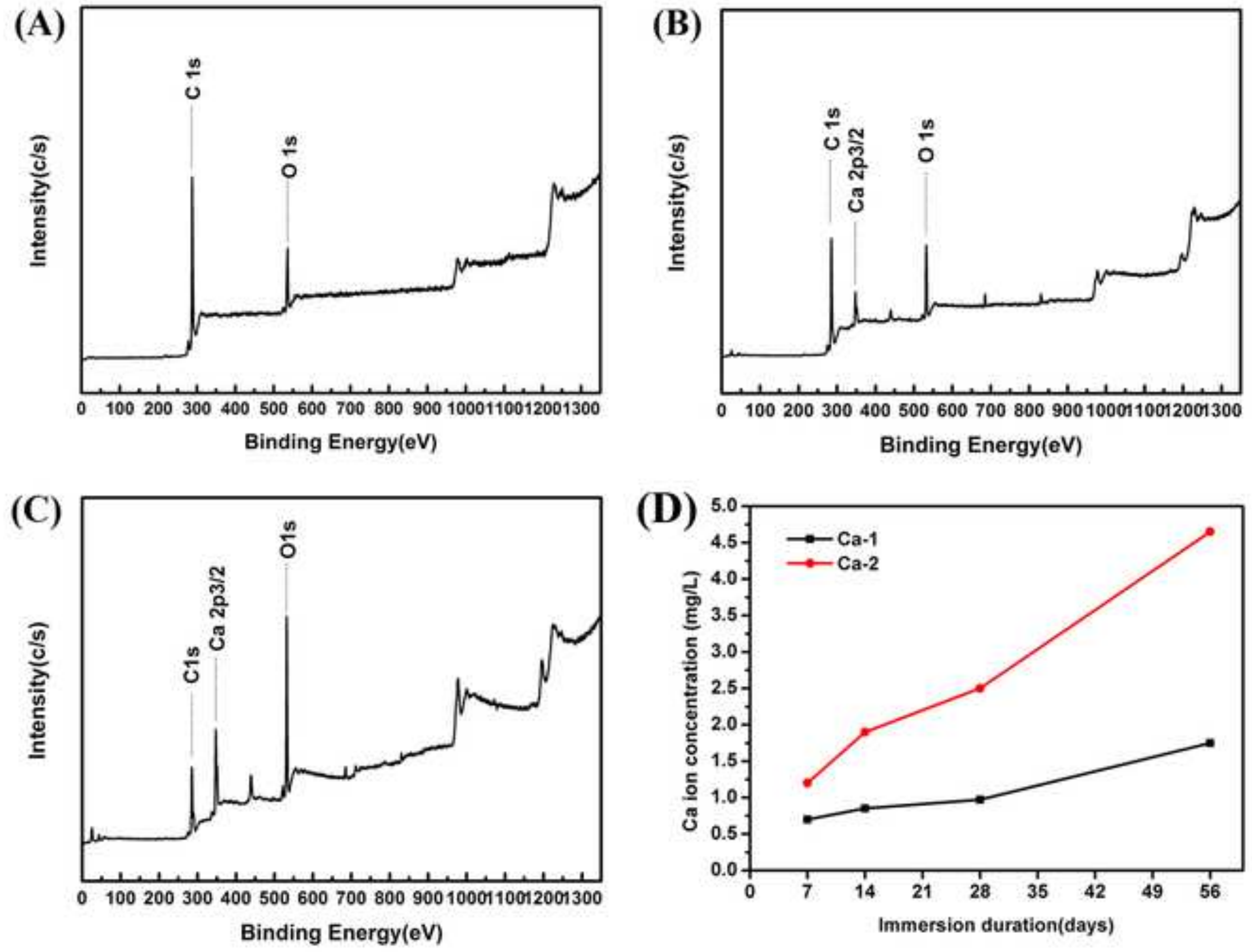


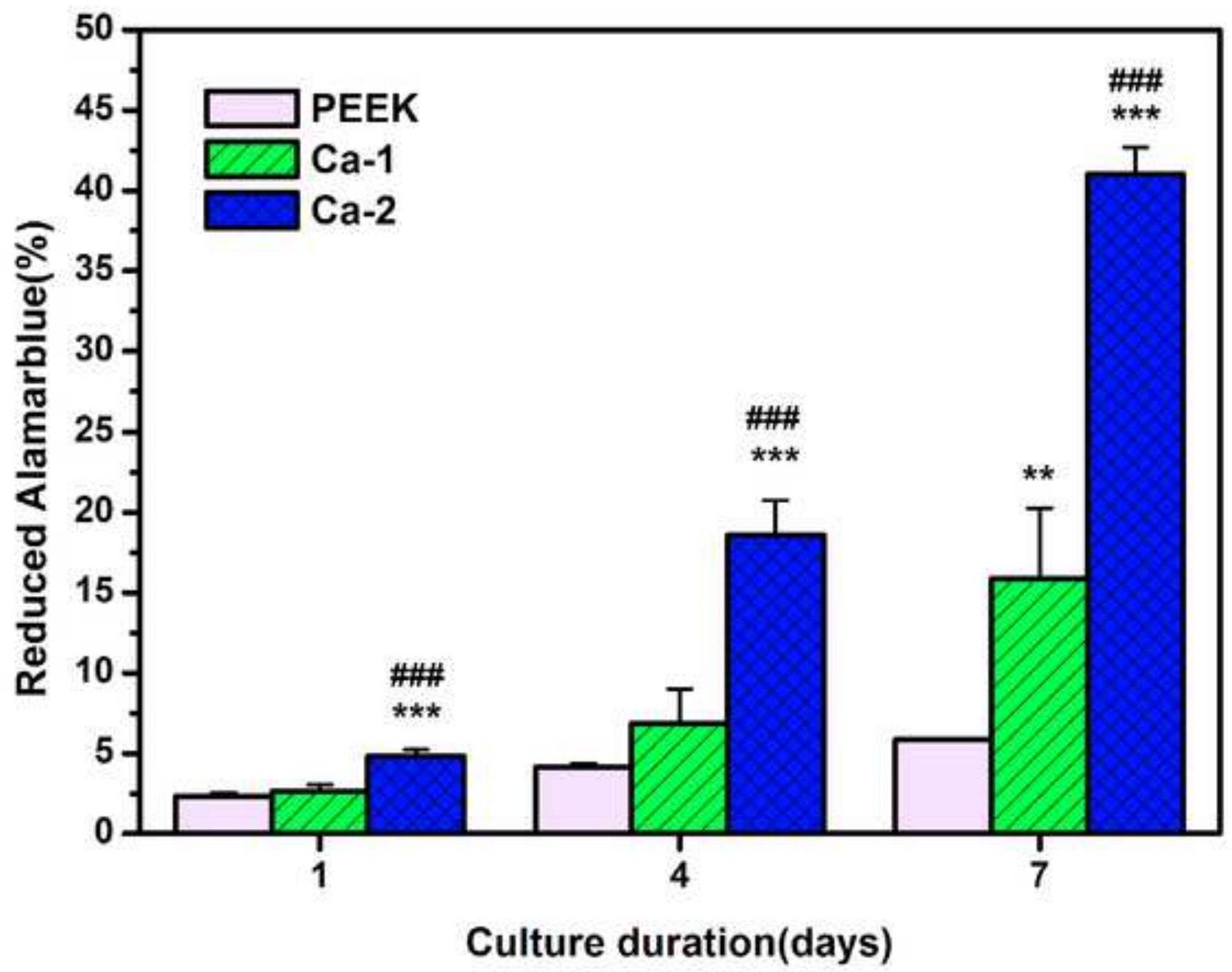




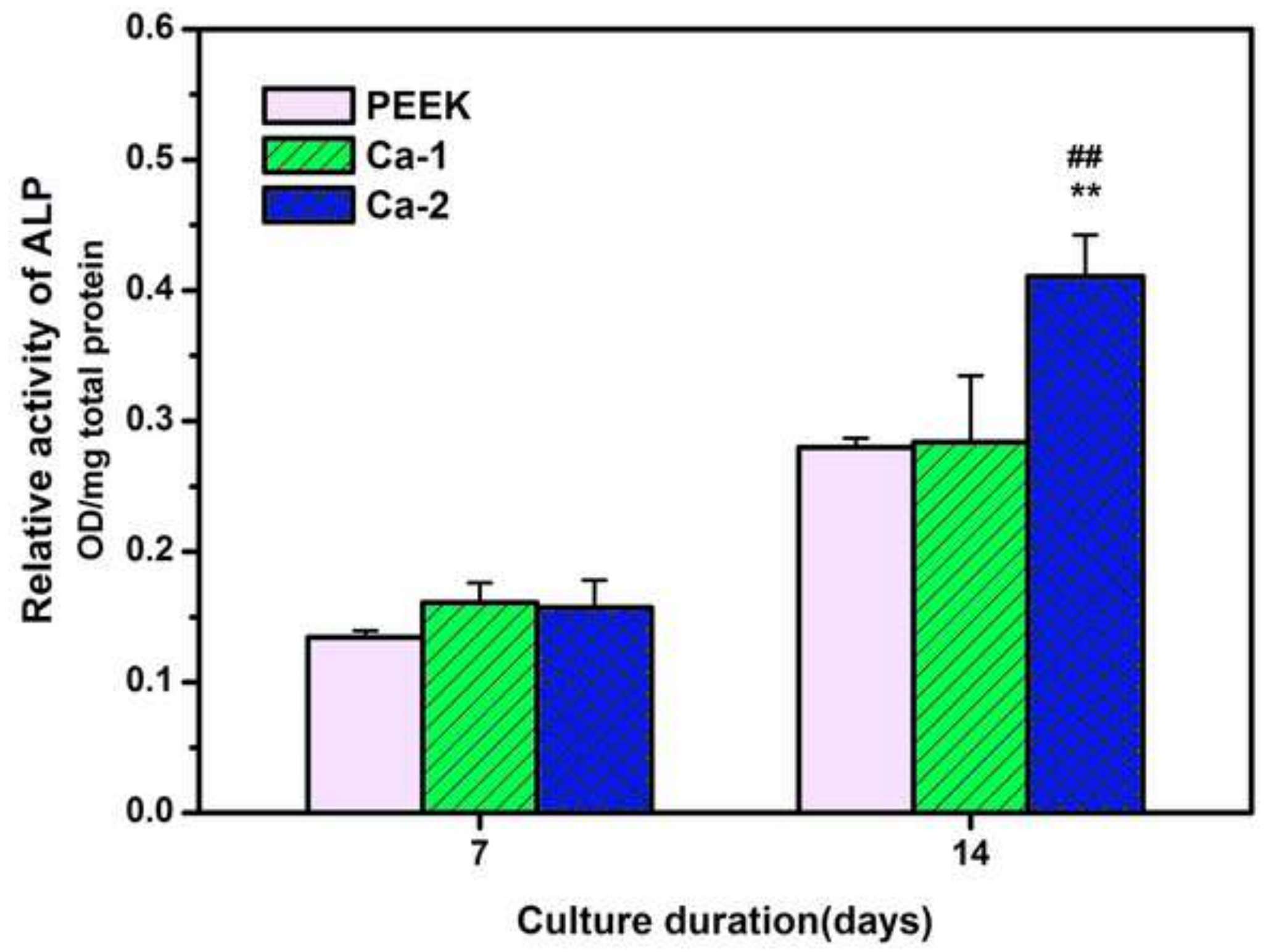



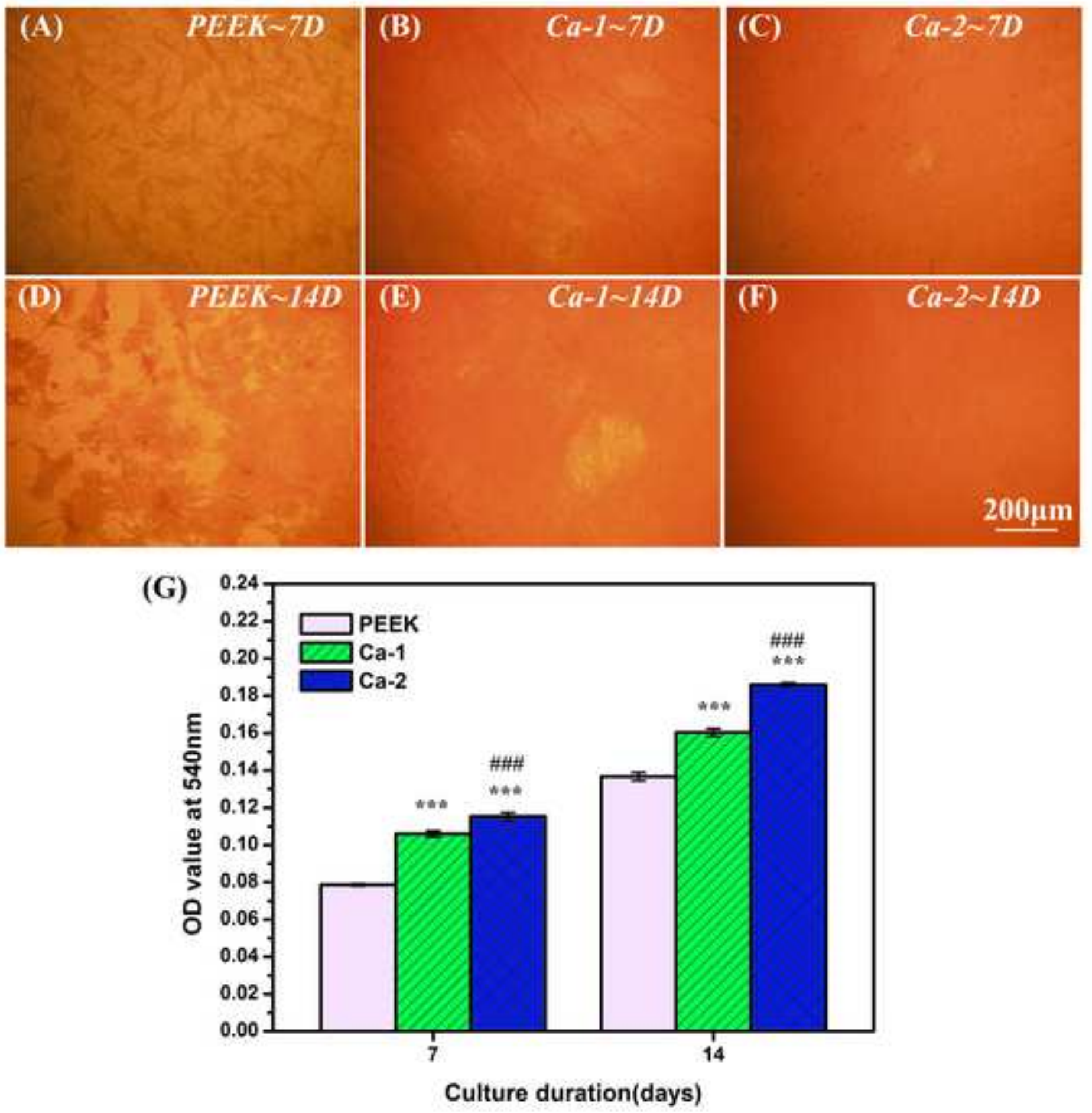
Table 1. Main conditions used in calcium plasma immersion ion implantation.

\begin{tabular}{lccc}
\hline & PEEK & Ca-1 & Ca-2 \\
\hline Cathodic arc pulse duration $(\mu \mathrm{s})$ & - & 500 & 500 \\
High voltage pulse duration $(\mu \mathrm{s})$ & - & 500 & 500 \\
Pulsing frequency $(\mathrm{Hz})$ & - & 7 & 7 \\
Implantation voltage $(\mathrm{kV})$ & - & 15 & 30 \\
Implantation time $(\mathrm{min})$ & - & 60 & 60 \\
Pulsed arc current $(\mathrm{A})$ & - & 0.2 & 0.2 \\
Pulsed arc voltage $(\mathrm{kV})$ & - & 13.1 & 13.1 \\
\hline
\end{tabular}

